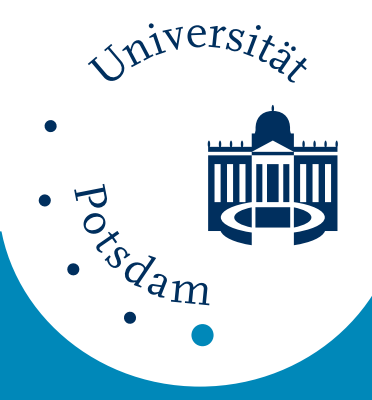

Universität Potsdam

Norbert Seehafer, Jörg Schumacher

Bifurcation analysis of the plane sheet pinch

NLD Preprints ; 44 
[To appear in Phys. Plasmas, scheduled June 1998]

\title{
Resistivity profile and instability of the plane sheet pinch
}

\author{
N. Seehafer ${ }^{1}$ AND J. SChumaCher ${ }^{2}$ \\ ${ }^{1}$ Institut für Physik, Universität Potsdam, PF 601553, D-14415 Potsdam, Germany \\ ${ }^{2}$ Fachbereich Physik, Philipps-Universität Marburg, D-35032 Marburg, Germany
}

March 9, 1998

\begin{abstract}
The stability of the quiescent ground state of an incompressible, viscous and electrically conducting fluid sheet, bounded by stress-free parallel planes and driven by an external electric field tangential to the boundaries, is studied numerically. The electrical conductivity varies as $\cosh ^{2}\left(x_{1} / a\right)$, where $x_{1}$ is the cross-sheet coordinate and $a$ is the half width of a current layer centered about the midplane of the sheet. For $a \lesssim 0.4$ the ground state is unstable to disturbances whose wavelengths parallel to the sheet lie between lower and upper bounds depending on the value of $a$ and on the Hartmann number. Asymmetry of the configuration with respect to the midplane of the sheet, modelled by the addition of an externally imposed constant magnetic field to a symmetric equilibrium field, acts as a stabilizing factor.
\end{abstract}

PACS Numbers 52.30.-q, 47.65.+a, 95.30.Qd 


\section{INTRODUCTION}

The equilibrium states of electrically conducting fluids or plasmas have been a subject of intense study for a long time, motivated in particular by the interest in controlled thermonuclear fusion, as well as that in space and astrophysical phenomena such as plasma loops in the solar corona. If high temperatures prohibit solid walls, a conducting fluid can be held together by the action of an electric current passing through it with the pressure gradients being balanced by the Lorentz force. The resultant configuration is known as a pinch.

Pinch configurations are subject to various instabilities ${ }^{1,2}$. Of special interest here are the tearing modes, which belong to the class of finite-resistivity instabilities. By destroying magnetic surfaces, they can shorten the confinement time of fusion plasmas. Tearing modes represent one of the basic mechanisms for magnetic reconnection and are also thought to play a role in the explosive release of magnetic energy in space and astrophysical plasmas ( e.g., substorms in the terrestrial magnetosphere and solar flares $\left.{ }^{3}\right)$.

In a plane sheet geometry, the pinch with the fluid at rest is absolutely stable if the electrical conductivity is infinite - the case of ideal magnetohydrodynamics (MHD) - but may be destabilized by resistivity. A systematic theory of the resistive instability of the plane sheet pinch was developed by Furth et $a l^{4}{ }^{4}$, who used a boundary layer approach, dividing the sheet into a narrow inner resistive layer and outer regions with perfect conductivity (accounts of this approach may also be found in Refs. $\left.{ }^{1,2,5}\right)$. Numerical confirmation of the analytical results of Furth et al. was obtained by Wesson ${ }^{6}$, Schnack and Killeen ${ }^{7}$, and Steinolfson and Van Hoven ${ }^{8}$, who studied the basic equations of Furth et al. without making the boundary-layer approximation. These basic equations are the general MHD equations, but with viscosity neglected. As noted by Dahlburg et al. ${ }^{9}$, the stability boundaries of the sheet pinch are determined by the Hartmann number $\mathrm{Ha}$. A similar result for 
the cylindrical pinch was proven by Montgomery ${ }^{10}$. $\mathrm{Ha}$ is the geometric mean of two Reynolds-like numbers, one being kinetic and the other magnetic. These do not influence the stability boundaries independently, but only in combination in the Hartmann number. Thus, all calculations of stability boundaries in which viscosity is neglected pertain to the limit $\mathrm{Ha} \rightarrow \infty$ and the stability boundaries obtained are independent of the remaining (magnetic) Reynolds-like number.

A recent study ${ }^{11}$ has been done on the MHD equations without any boundarylayer approximation and with viscosity taken into account, in a voltage-driven incompressible sheet pinch with spatially and temporally uniform kinematic viscosity and magnetic diffusivity, as well with impenetrable stress-free boundaries. It is found that the quiescent ground state (in which the current density is uniform and the magnetic field profile across the sheet is linear) remains stable, no matter how strong the driving electric field. This contrasts with results of Shan, Montgomery, and Chen ${ }^{12}$ for the voltage-driven cylindrical pinch. These authors observed, as an externally applied electric field was raised, transitions first to stationary states with flow and eventually to turbulent states. The situation is reminiscent of the difference between plane and rotating hydrodynamic Couette flow $^{13}$. Specifically, for the plane Couette flow - the flow between infinite parallel planes with one moving boundary - the ground state with a linear shear flow profile is stable. For the rotating Couette flow - the flow between differentially rotating coaxial cylinders of which the inner one rotates faster - the laminar ground state becomes unstable if the rotation rates of the two cylinders are sufficiently different.

In the present paper the sheet-pinch study of Ref. ${ }^{11}$ is extended to the case of electrical conductivity varying across the sheet. This results in the profiles of the equilibrium magnetic field deviating from linear behaviour. In particular, the conductivity profile may be chosen such that the magnetic-field and/or current profile has inflection points. Inflection points in the velocity profile are known to 
be necessary (but not sufficient) for the instability of inviscid plane shear flows ${ }^{13}$. With respect to the plane sheet pinch, Dahlburg et al. ${ }^{9}$ observed instability to be associated with inflection points in the current profile. These authors along with Saramito and Maschke ${ }^{14,15}$ in related work, studied quasi-equilibria, namely, states with a nonuniform current density in a fluid with uniform resistivity (such states decay resistively). The present study investigates exact pinch equilibria, driven by an external electric field tangential to the boundary planes. Our main concern is with the influence of the cross-sheet resistivity profile on the stability boundaries. The resistivity profile determines the current profile and the magnetic field profile can then still be modified by an externally imposed constant field. Given the crosssheet profiles, the stability boundaries are determined by the Hartmann number. We consider a configuration with a current sheet centered about the midplane of the sheet and study in detail how the degree of current concentration in the sheet center influences the stability properties. We also study the influence of asymmetries in the magnetic field profile, introduced by adding an externally imposed field to the self-consistently supported one.

In Sec. II we outline the governing equations and define the boundary conditions and the equilibrium state. Then, in Sec. III we describe the method of stability analysis and discuss some general properties of the problem. In Sec. IV we present and discuss our numerical results. In Sec. V, finally, a brief summary and an outlook are given.

\section{BASIC EQUATIONS, BOUNDARY CONDITIONS, AND EQUILIB- RIUM}

We use the nonrelativistic, incompressible MHD equations,

$$
\rho\left(\frac{\partial \mathbf{v}}{\partial t}+(\mathbf{v} \cdot \nabla) \mathbf{v}\right)=\rho \nu \nabla^{2} \mathbf{v}-\nabla p+\mathbf{J} \times \mathbf{B}
$$




$$
\begin{aligned}
& \frac{\partial \mathbf{B}}{\partial t}=-\nabla \times\left(\eta \mu_{0} \mathbf{J}-\mathbf{v} \times \mathbf{B}\right), \\
& \nabla \cdot \mathbf{v}=0, \quad \nabla \cdot \mathbf{B}=0,
\end{aligned}
$$

where $\mathbf{v}$ is the fluid velocity, $\mathbf{B}$ the magnetic induction, $\mathbf{J}$ the electric current density $\left(=\nabla \times \mathbf{B} / \mu_{0}, \mu_{0}\right.$ denoting the magnetic permeability in a vacuum), $\rho$ the mass density, $p$ the thermal pressure, $\nu$ the kinematic viscosity, and $\eta$ the magnetic diffusivity $\left[\left(\mu_{0} \eta\right)^{-1}\right.$ is the electrical conductivity]. No externally applied force appears in Eq. (1). While $\rho$ and $\nu$ are assumed constant, $\eta$ is allowed to vary spatially (but not temporally):

$\eta(\mathbf{x})=\eta_{0} \tilde{\eta}(\mathbf{x})$

where $\eta_{0}$ is a dimensional constant and $\tilde{\eta}(\mathbf{x})$ a dimensionless function of position.

Let $L=L_{1}$ and $B_{0}$ denote arbitrary units of length and magnetic induction. Writing $v_{A}=B_{0} / \sqrt{\mu_{0} \rho}$ for the Alfvén velocity corresponding to $B_{0}$, we transform to dimensionless quantities according to

$$
\begin{array}{r}
\mathbf{x} / L \rightarrow \mathbf{x}, \mathbf{B} / B_{0} \rightarrow \mathbf{B}, \mathbf{v} / v_{A} \rightarrow \mathbf{v}, t / \frac{L}{v_{A}} \rightarrow t, \\
p / \rho v_{A}^{2} \rightarrow p, \mathbf{J} / \frac{B_{0}}{\mu_{0} L} \rightarrow \mathbf{J}, \quad \mathbf{E} / B_{0} v_{A} \rightarrow \mathbf{E} .
\end{array}
$$

$\mathbf{E}$ is the electric field. Eqs. (1) and (2) then become

$$
\begin{aligned}
& \frac{\partial \mathbf{v}}{\partial t}=-(\mathbf{v} \cdot \nabla) \mathbf{v}+M^{-1} \nabla^{2} \mathbf{v}-\nabla p+\mathbf{J} \times \mathbf{B}, \\
& \frac{\partial \mathbf{B}}{\partial t}=-\nabla \times\left(S^{-1} \tilde{\eta} \mathbf{J}-\mathbf{v} \times \mathbf{B}\right),
\end{aligned}
$$

where

$$
M=\frac{v_{A} L}{\nu} \text { and } S=\frac{v_{A} L}{\eta_{0}}
$$

are Reynolds-like numbers based on the Alfvén velocity: $S$ is the Lundquist number and $M$ its viscous analogue. The dimensionless Ohm's law becomes 
$S^{-1} \tilde{\eta} \mathbf{J}=\mathbf{E}+\mathbf{v} \times \mathbf{B}$.

We use Cartesian coordinates $x_{1}, x_{2}, x_{3}$ and consider our magnetofluid in the slab $0<x_{1}<1$. In the $x_{2}$ and $x_{3}$ directions periodic boundary conditions are assumed. The geometry of the slab configuration is shown in Fig. 1.

The boundary planes are assumed to be impenetrable and stress-free, i.e.,

$v_{1}=\frac{\partial v_{2}}{\partial x_{1}}=\frac{\partial v_{3}}{\partial x_{1}}=0 \quad$ at $x_{1}=0,1$

The system is driven by an electric field of strength $E^{*}$ in the $x_{3}$ direction, which can be prescribed only on the boundary. We further assume that there is no magnetic flux through the boundary,

$B_{1}=0 \quad$ at $x_{1}=0,1$.

Conditions (10) and (11) imply that the tangential components of $\mathbf{v} \times \mathbf{B}$ on the boundary planes vanish, so that according to Eq. (9)

$J_{2}=0, \quad J_{3}=\frac{E^{*} S}{\tilde{\eta}_{b}} \quad$ at $x_{1}=0,1$

where $\tilde{\eta}_{b}$ is the value of $\tilde{\eta}$ on the boundaries. The boundary conditions for the tangential components of $\mathbf{B}$ then become $(\mathbf{J}=\nabla \times \mathbf{B}$ in the dimensionless units $)$ $\frac{\partial B_{2}}{\partial x_{1}}=\frac{E^{*} S}{\tilde{\eta}_{b}}, \quad \frac{\partial B_{3}}{\partial x_{1}}=0 \quad$ at $x_{1}=0,1$.

A discussion of these boundary conditions is found in Ref. ${ }^{11}$.

Any stationary state with the fluid at rest has to satisfy the equations

$$
\begin{array}{r}
-\nabla p+\mathbf{J} \times \mathbf{B}=\mathbf{0}, \\
\nabla \times(\tilde{\eta} \mathbf{J})=\mathbf{0} .
\end{array}
$$

For $\tilde{\eta}$ depending only on the cross-sheet coordinate $x_{1}$, Eqs. (14), (15) and the boundary conditions are then satisfied with 


$$
\begin{aligned}
\mathbf{J} & =\mathbf{J}^{e}=\left(0,0, \tilde{\eta}^{-1} E^{*} S\right) \\
\mathbf{B} & =\mathbf{B}^{e}=\left(0, E^{*} S I_{0}\left(x_{1}\right)+\overline{B_{2}^{e}}, \overline{B_{3}^{e}}\right), \\
p & =p^{e}=-\frac{\mathbf{B}^{e 2}}{2}
\end{aligned}
$$

where overbars denote spatial averages and $I_{0}\left(x_{1}\right)=\int \tilde{\eta}^{-1} \mathrm{~d} x_{1}-\overline{\int \tilde{\eta}^{-1} \mathrm{~d} x_{1}}$.

We use the diffusivity profile

$\tilde{\eta}=\cosh ^{2}\left[\left(x_{1}-0.5\right) / a\right]$

where $a$ is the current sheet half width. The magnetic field unit, $B_{0}$, is chosen in such a way that, in the case of $\overline{B_{2}^{e}}=0,\left|B_{2}^{e}\right|=1$ on the boundary planes. This fixes the value of $E^{*}$ such that

$E^{*}=\left[S I_{0}(1)\right]^{-1}$,

and then the equilibrium magnetic field can be written as

$B_{2}^{e}=[\tanh (1 / 2 a)]^{-1} \tanh \left[\left(x_{1}-0.5\right) / a\right]+\overline{B_{2}^{e}}$.

This is the frequently studied Harris ${ }^{16}$ sheet.

\section{STABILITY ANALYSIS}

The system of Eqs. (3), (6), and (7) has been studied by means of a pseudospectral method in Fourier space. The treatment is analogous to that of the case with spatially uniform magnetic diffusivity in Seehafer et al. ${ }^{11}$ and details may be found there. As in the case of uniform $\eta$, the spatial means of $v_{2}, v_{3}, B_{2}$, and $B_{3}$ are independent of time. Without loss of generality we have restricted ourselves to the case of $\overline{v_{2}}=\overline{v_{3}}=0$, since the mean flow can be removed by a Galilean transformation. The mean values $\overline{B_{2}^{e}}$ and $\overline{B_{3}^{e}}$ are considered as parameters.

We use the notation

$$
P=p+\frac{1}{2} \mathbf{B}^{2}, \quad \mathbf{b}=\mathbf{B}-\mathbf{B}^{e}, \quad \mathbf{j}=\mathbf{J}-\mathbf{J}^{e}
$$


where $\mathbf{v}$ and $\mathbf{b}$ are our dynamical variables, for which the complete boundary conditions are as follows:

$v_{1}=\frac{\partial v_{2}}{\partial x_{1}}=\frac{\partial v_{3}}{\partial x_{1}}=b_{1}=\frac{\partial b_{2}}{\partial x_{1}}=\frac{\partial b_{3}}{\partial x_{1}}=0 \quad$ at $x_{1}=0,1$.

We Fourier expand into modes $\sim \exp \left\{i\left(k_{2} x_{2}+k_{3} x_{3}\right)\right\}$ in the $x_{2}$ and $x_{3}$ directions. Let $v_{i \mathbf{k}}, b_{i \mathbf{k}}$, and $j_{i \mathbf{k}}$ denote the Fourier coefficients of $v_{i}, b_{i}$, and $j_{i}$, respectively, for wavenumber $\mathbf{k}=\left(k_{2}, k_{3}\right)$. Linearizing about the static equilibrium, Eqs. (6) and (7) become

$$
\begin{aligned}
& \dot{v}_{1 \mathbf{k}}=-P_{\mathbf{k}}^{\prime}-M^{-1}\left(\mathbf{k}^{2}-D^{2}\right) v_{1 \mathbf{k}}+i F_{\mathbf{k}} b_{1 \mathbf{k}}, \\
& \dot{v}_{2 \mathbf{k}}=-i k_{2} P_{\mathbf{k}}-M^{-1}\left(\mathbf{k}^{2}-D^{2}\right) v_{2 \mathbf{k}}+i F_{\mathbf{k}} b_{2 \mathbf{k}}+\left(B_{2}^{e}\right)^{\prime} b_{1 \mathbf{k}}, \\
& \dot{v}_{3 \mathbf{k}}=-i k_{3} P_{\mathbf{k}}-M^{-1}\left(\mathbf{k}^{2}-D^{2}\right) v_{3 \mathbf{k}}+i F_{\mathbf{k}} b_{3 \mathbf{k}} \\
& \dot{b}_{1 \mathbf{k}}=i F_{\mathbf{k}} v_{1 \mathbf{k}}-S^{-1}\left[i k_{2} \tilde{\eta} j_{3 \mathbf{k}}-i k_{3} \tilde{\eta} j_{2 \mathbf{k}}\right] \\
& \dot{b}_{2 \mathbf{k}}=i F_{\mathbf{k}} v_{2 \mathbf{k}}-\left(B_{2}^{e}\right)^{\prime} v_{1 \mathbf{k}}-S^{-1}\left[i k_{3} \tilde{\eta} j_{1 \mathbf{k}}-\left(\tilde{\eta} j_{3 \mathbf{k}}\right)^{\prime}\right], \\
& \dot{b}_{3 \mathbf{k}}=i F_{\mathbf{k}} v_{3 \mathbf{k}}-S^{-1}\left[\left(\tilde{\eta} j_{2 \mathbf{k}}\right)^{\prime}-i k_{2} \tilde{\eta} j_{1 \mathbf{k}}\right],
\end{aligned}
$$

where $F_{\mathbf{k}}=k_{2} B_{2}^{e}+k_{3} \overline{B_{3}^{e}}$ and $D \equiv{ }^{\prime} \equiv \partial / \partial x_{1}$. Both equilibrium magnetic field components, $B_{2}^{e}$ and $\overline{B_{3}^{e}}$, are combined in one single profile function, $F_{\mathbf{k}}\left(x_{1}\right)$.

The special modes with $k_{2}=0$ cannot become unstable since $B_{2}^{e}$ does not enter the equations for them; they thus always behave as if $E^{*}=0$ [cf. Eq. (17)]. For modes with $k_{2} \neq 0$ a constant field component $\overline{B_{3}^{e}}$ in the sheetwise direction parallel to the driving electric field acts in the same way as a constant field component $\overline{B_{2}^{e}}\left(=\left(k_{3} / k_{2}\right) \overline{B_{3}^{e}}\right)$. It does not lead to oscillations as incorrectly argued in a former article ${ }^{17}$ where a Squire's theorem for a voltage-driven sheet pinch is proved, stating that for each unstable three-dimensional perturbation there exists a more unstable two-dimensional one (with vectors $\mathbf{v}$ and $\mathbf{b}$ lying completely in the $x_{1}-x_{2}$ plane and having no $x_{3}$ dependence). Also, as the Reynolds-like numbers $M$ and $S$ are raised from small values, two-dimensional perturbations become unstable first. This proof is valid for $\overline{B_{3}^{e}}=0$. An immediate implication is that an unstable 
equilibrium with $\overline{B_{3}^{e}}=0$ can never be stabilized by adding a nonvanishing $\overline{B_{3}^{e}}$. As is seen from the definition of the profile function $F_{\mathbf{k}}, \overline{B_{3}^{e}}$ influences only the stability of modes with $k_{3} \neq 0$ and does not influence the (in the absence of a $\overline{B_{3}^{e}}$ ) most unstable modes, for which $k_{3}=0$. Therefore the field component $\overline{B_{3}^{e}}$ cannot increase the global stability. It is possible, however, that the addition of $\overline{B_{3}^{e}}$ makes an equilibrium less stable (see Sec. IV B).

To determine the stability of the Harris sheet equilibrium, with $\tilde{\eta}$ and $B_{2}^{e}$ given by Eqs. (19) and (21), the eigenvalues of the Jacobian matrix of our system at the equilibrium (i.e., the eigenvalues of the linear operator on the right-hand side of the system (24)) have been calculated. Since a nonvanishing $\overline{B_{3}^{e}}$ can be formally transformed into a $\overline{B_{2}^{e}}$ and for vanishing $\overline{B_{3}^{e}}$ the Squire's theorem is valid, the calculations have been restricted to the case of $\overline{B_{3}^{e}}=0$ and to two spatial dimensions. The remaining current density component is $j_{3 \mathbf{k}}=b_{2 \mathbf{k}}^{\prime}-i k_{2} b_{1 \mathbf{k}}$.

We have used expansions of $v_{1}$ and $b_{1}$ in pure sine series and of $v_{2}$ and $b_{2}$ in pure cosine series with respect to $x_{1}$, in correspondence with the boundary conditions. The numerical calculations were made with 64 (and partially with 128) collocation points in the cross-sheet $\left(x_{1}\right)$ direction and just one wave number, $k_{2}=\mp 2 \pi / L_{2}$, in the $x_{2}$ direction.

At the stability threshold two identical real eigenvalues were always observed to pass through zero. The fact that unstable eigensolutions are non-oscillatory has been known since the paper of Furth et al. ${ }^{4}$ The multiplicity of the eigenvalues is obviously due to the $O(2)$ symmetry of the problem with respect to the $x_{2}$ direction: The translational symmetry in this direction combined with the periodic boundary conditions gives a circle symmetry $S O(2)$, which together with the symmetry to reflections in the planes $x_{2}=$ const. leads to an $O(2)$ symmetry. $O(2)$ symmetry, however, always forces the eigenvalues to have multiplicity two $^{18}$, simply because with each eigenmode $\sim \exp \{i k x\}$ there is another, 'reflected' 
eigenmode $\sim \exp \{-i k x\}$ for the same eigenvalue, and the two modes are linearly independent. (This argument does not apply to modes with $k_{2}=0$.)

Since the critical eigenvalues are real, one has $\dot{\mathbf{v}}=\dot{\mathbf{b}}=\mathbf{0}$ at the stability threshold. In this case the rescaling

$M^{-1 / 2} v_{j \mathbf{k}} \rightarrow v_{j \mathbf{k}}, \quad S^{-1 / 2} b_{j \mathbf{k}} \rightarrow b_{j \mathbf{k}}, \quad S^{-1 / 2} P_{\mathbf{k}} \rightarrow P_{\mathbf{k}}$

in Eqs. (24) leads to equations (valid at the threshold) in which $M$ and $S$ do not occur separately but only arise when combined in the Hartmann number $H a=(M S)^{1 / 2}$, which thus determines the stability boundary ${ }^{9,10,19}$ — together with the current sheet half width $a$, the constant magnetic field component $\overline{B_{2}^{e}}$ (both contained in the equilibrium profile $F_{\mathbf{k}}$ ), and the wave number $k_{2}$ of the perturbation.

\section{RESULTS AND DISCUSSION}

\section{A. Equilibrium with $\overline{B_{2}^{e}}=0$}

In this subsection we present numerical results for the case of $\overline{B_{2}^{e}}=0$. We have studied equilibria with $a \geq 0.1$. The minimum Hartmann number necessary for instability is then $H a=64.6$ (reached for the parameters $a=0.1, L_{2}=2.4$ ). Figure 2 shows, for different values of the Hartmann number, numerically determined stability boundaries in the $a-L_{2}$ plane. Each stability boundary consists of two branches - an upper one and a lower one- with the unstable region lying between both branches (to the left of the total, parabola-like stability curve). The equilibrium is found to be stable for $a>a_{c}$ and unstable for $a<a_{c}$, where $a_{c}$ is a critical current sheet half width. The parameter $a_{c}$ depends on $H a$, but $a_{c} \lesssim 0.41$ (see below). In the case of instability, the wavelengths of the unstable modes in the $x_{2}$ direction lie between lower and upper bounds depending on $a$. As $a$ approaches $a_{c}$, the unstable wavelength interval shrinks to zero. The previously studied case 
of a spatially uniform resistivity and a linear magnetic field profile ${ }^{11}$ corresponds to the limit $a \rightarrow \infty$.

Figure 3 shows magnetic field lines and velocity stream lines of an unstable mode, for the parameters $L_{2}=3, a=0.15$, and $H a=100$. The typical tearing-mode pattern is clearly visible. Null points of $\mathbf{B}$ - a chain of $O$ points and $X$ points in alternating order, the $O$ points being centers of magnetic islands - coincide with stagnation points of the flow, which is organized in convection-like cells.

The magnetic field profile has an inflection point in the center of the sheet (the inflection points of the magnetic field profile simply correspond to extrema of the current profile) while the current profile has two inflection points, one on either side of the midplane $x_{1}=0.5$. We also note that for values of $a$ well above 0.41 , the two inflection points in the current profile are still situated well within the sheet. So their presence in the sheet (combined of course with a sufficiently strong driving to overcome dissipation) is not enough for instability, but the current must be sufficiently concentrated in the sheet center. The reverse holds if the current is unidirectional and sufficiently concentrated. In this case, the current profile must have inflection points within the sheet.

Traditional boundary-layer theory of the sheet pinch $^{4}$ finds tearing modes to be stable for large and unstable for small wavenumbers $k_{2}$. If the equilibrium is just the Harris sheet, $k_{2} a \leq 1$ is the condition for instability. This result refers to infinitely distant walls or very small values of $a$, lying in a region to the left of that covered in Fig. 2. For our smallest value of $a, 0.1$, we have found a critical lower wavelength of $L_{2} \approx 0.63$ (see the lower branch of the curve for $H a=10^{7}$ in Fig. 2), corresponding to $k_{2} a \approx(2 \pi / 0.63) 0.1 \approx 1$, in agreement with the condition from the boundary-layer analysis.

In Appendix $G$ of Furth et $a .^{4}$ the influence of walls at a finite distance is also considered. In that paper the current-sheet half width $a$ is used as the unit of length, so that instead of $a$ the distance of the walls has to be varied. By modifying 
the solution in the outer, perfectly conducting regions, the walls have a stabilizing influence. If, in our units, $a$ approaches the value 1/2.4 from below, the minimum wavelength for instability tends to infinity, so that for $a>1 / 2.4$ absolute stability is achieved. Also, the lower branches of the curves in Fig. 2 steeply increase as $a$ approaches $a_{c}$, but the upper critical wavelengths simultaneously decrease sharply such that the marginal stability curves in the $a$-wavelength plane have turning points at $a=a_{c}$. An upper critical wavelength for instability is not reported in Furth et al., and has not been observed in subsequent studies using the same basic equations. Our calculations with very high Hartmann numbers indicate that $a \approx 0.41(\ldots 0.42)$ is a limiting value for the location of the turning point (see Fig. 2 for $H a=10^{7}$ ), which is thus not shifted to larger values for $H a \rightarrow \infty$. This conclusion is supported by the result in Furth et al., namely, the stability for $a>1 / 2.4$, as well as by numerical results of Saramito and Maschke ${ }^{14}$, who found stability of the quasi-stationary ( $\eta$ was assumed uniform) Harris equilibrium for $a^{-1} \lesssim 2.5$

With respect to the treatment in Appendix G of Furth et al., we note that an 'infinite-conductivity' equation is solved there to determine the lower critical wavelength (or upper critical wave number, respectively) for instability and its dependence on the distance of the walls. However, in the case of marginal stability this equation is valid without the division into resistive and ideal regions. Specifically, in the paper of Furth et al. the 'infinite-conductivity' equation, Eq. (G.1), is seen to follow from Eq. (17) (of Ref. ${ }^{4}$ ) if the growth rate of the instability is set equal to zero. Equation (17) is one of the linearized equations determining the stability and is, except for the neglect of viscosity, still general. This fact was used by Shivamoggi ${ }^{20}$ to study the effect of the walls without the division into separate regions (but also neglecting viscosity). The only remaining approximation is then the neglect of viscosity, which amounts to studying the limit $H a \rightarrow \infty$. One can be rather confident, therefore, that the limit $a_{c} \leq 1 / 2.4=0.417$ holds. 
We include some more speculative considerations in the following. It is well known that the wavelength of a perturbation parallel to the equilibrium field has to exceed a critical value in order for the tearing mode to lead to instability. The driving mechanism of the instability is the mutual attraction of parallel currents, and the separate pinches into which the sheet breaks have to be sufficiently long compared to the current-sheet thickness. This leads to the lower branches of the stability boundaries in Fig. 2. On the other hand, if the magnetic islands and the convection cells become too long, diffusion across the sheet - Ohmic and viscousmight become important and quench the instability. This would explain the upper branches in Fig. 2. Instead of thinking of the islands becoming longer, one may equivalently imagine that the walls come closer to the midplane of the sheet. The latter enhances the diffusive damping of perturbations (due to steeper gradients with respect to $x_{1}$ ), in a similar way as increased values of the diffusivities would. The Hartmann number necessary for instability then becomes larger, leading to the upper branches in Fig. 2. Also, increasing the current-sheet half width $a$ acts as if the walls would come closer (if we had chosen $a$ as the unit of length, we would have to vary the distance of the walls instead of varying $a$ ). This can explain the decrease of the upper critical wavelength with increasing $a$. It does not explain yet why the turning point where lower and upper branches meet is not shifted to larger and larger values of $a$ as $H a \rightarrow \infty$. Seemingly, for walls which are too close or current profiles which are too flat the basic driving mechanism of the tearing instability no longer works.

\section{B. Equilibrium with $\overline{B_{2}^{e}} \neq 0$}

In this subsection the influence of an asymmetry of $B_{2}^{e}$ on the stability is studied. For this purpose we vary $\overline{B_{2}^{e}}$ in the interval [0,1] [cf. Eq. (21)]. Figure 4 shows, for $H a=10^{3}$ and $H a=10^{4}$, respectively, the stability boundaries for different 
values of $\overline{B_{2}^{e}}$. Again the unstable regions lie to the left of the respective curves. In the case $\overline{B_{2}^{e}}=1$ there is no surface $F_{\mathbf{k}}=0$ or no reversal of the equilibrium field in the sheet, so that magnetic islands cannot form and the equilibrium remains stable. The larger the constant part of the profile, the more stable the equilibrium becomes, i.e., the smaller the unstable region in the $a-L_{2}$ plane between the two branches of the stability boundary. As $\overline{B_{2}^{e}} \rightarrow 1$, the unstable wavelength interval shrinks to zero. Figure 5 shows magnetic field lines and velocity stream lines for a typical asymmetric case.

Let $\Theta_{0}$ denote the ratio of the maximum self-consistently supported magnetic field to the externally imposed magnetic field, i.e., $\Theta_{0}=1 / \overline{B_{2}^{e}}$ in our dimensionless units. Figure 6 shows stability boundaries in the $H a-\Theta_{0}$ plane, calculated for three different parameter sets $\left(a, L_{2}\right)$. The curves shown here are global stability boundaries, i.e., the envelopes of all the modal stability boundaries in the respective cases. Quiescent states with high Hartmann numbers (with strong currents) are possible, provided $\Theta_{0}$ is small enough. Diamonds in Fig. 6 correspond to parameters $\left(H a, \overline{B_{2}^{e}}\right)$ for which stability boundaries are shown in Fig. 4.

It is now possible that the addition of a $\overline{B_{3}^{e}}$ makes an asymmetric equilibrium less stable or even a stable one unstable. Let $\tilde{B}_{2}^{e}\left(x_{1}\right)$ be a symmetric profile admitting unstable two-dimensional perturbations, and let the constant $\overline{B_{2}^{e}}$ be chosen such that $B_{2}^{e}=\tilde{B}_{2}^{e}\left(x_{1}\right)+\overline{B_{2}^{e}}$ does not change sign within the sheet. The corresponding equilibrium is then stable to all two-dimensional perturbations, as seen before. However, for a given $\mathbf{k}=\left(k_{2}, k_{3}\right), \overline{B_{3}^{e}}$ can always be found such that $F_{\mathbf{k}}\left(x_{1}\right)$ changes sign within the sheet, for instance by the choice $\overline{B_{3}^{e}}=-\left(k_{2} / k_{3}\right) \overline{B_{2}^{e}}$. The stability problem [for the mode with $\mathbf{k}=\left(k_{2}, k_{3}\right)$ ] is then equivalent to the case with $B_{2}^{e}=\tilde{B}_{2}^{e}$ and $\overline{B_{3}^{e}}=0$. The Squire's transformation connects the threedimensional mode with wave vector $\mathbf{k}=\left(k_{2}, k_{3}\right)$ to a two-dimensional mode with wave number $\tilde{k_{2}}=\left(k_{2}^{2}+k_{3}^{2}\right)^{1 / 2}$, and the two modes are simultaneously stable or 
unstable. ${ }^{17}$ If the profile $\tilde{B}_{2}^{e}$ then admits growth of the two-dimensional mode with wave number $\tilde{k}_{2}$ (our numerical results show that this is certainly possible), the equilibrium is unstable to a three-dimensional perturbation, though it is stable to all two-dimensional perturbations. In such a case a Squire's theorem clearly cannot be valid.

At least for our special choice of the functional form of the resistivity profile, however, a Squire's theorem seems to be valid even with $\overline{B_{3}^{e}} \neq 0$ if the equilibrium $B_{2}^{e}$ is symmetric with respect to the midplane of the sheet. Namely, for a given $\mathbf{k}=\left(k_{2}, k_{3}\right), k_{3} \neq 0$, a nonvanishing $\overline{B_{3}^{e}}$ can be 'transformed' into a $\overline{B_{2}^{e}}$, so that the profile $B_{2}^{e}$ becomes asymmetric. For the new, asymmetric profile the threedimensional $\left(k_{3} \neq 0\right)$ perturbation is less unstable than some two-dimensional perturbation (Squire's theorem for a case with $\overline{B_{3}^{e}}=0$ ), which in turn is less unstable for the asymmetric than for the original, symmetric profile. So in the symmetric case $\overline{B_{3}^{e}}$ does not influence the global stability.

For a voltage-driven cylindrical pinch, with the plasma or magnetofluid occupying a full cylinder, the driving external electric field being axially directed, and the resistivity increasing with distance from the axis or being spatially uniform, it was found ${ }^{12,19,21}$ that the quiescent ground state can be stabilized by an externally imposed axial magnetic field component. However, in the case of the sheet pinch dc fields parallel to the driving electric field can never stabilize an equlibrium which is unstable in their absence (cf. Sec. III) and are completely irrelevant for the global stability if the configuration is symmetric to the midplane of the sheet. This difference seems to be connected with the fact that the instabilities of the cylindrical pinch are inherently three-dimensional, while the most unstable perturbations to the basic, symmetric sheet-pinch equlibrium are purely two-dimensional. 


\section{SUMMARY AND OUTLOOK}

The stability of the quiescent ground state of a Harris-type sheet-pinch equilibrium has been studied numerically by means of a pseudo-spectral method. The crosssheet resistivity profile was chosen such as to make the equilibrium an exact one. The stability of the quiescent ground state was found to be determined by the half width $a$ of the current layer centered about the midplane of the sheet, the Hartmann number, and the degree of asymmetry of the cross-sheet magnetic field profile. Only if the magnetic field profile is asymmetric can a dc field parallel to the driving electric field influence the global stability. Such a field can never stabilize an equilibrium which is unstable in its absence. For $a \lesssim 0.41$, the symmetric ground state is unstable to disturbances whose wavelengths parallel to the sheet lie between lower and upper bounds which depend on the value of $a$ and the Hartmann number. An upper critical wavelength for instability has not been noted before. Asymmetry of the configuration with respect to the midplane of the sheet, introduced by the addition of an externally imposed constant magnetic field, acts as a stabilizing factor (and can, if sufficiently strong, completely stabilize the pinch).

A stability analysis may be considered as a part of a bifurcation analysis, by which one tries to determine the set of possible time-asymptotic states, the attractors, for given values of the system parameters. The bifurcations from the Harris sheet have hitherto been studied for the case of two spatial dimensions ${ }^{14,15,22}$. The new time-asymptotic states are then of the tearing-mode type, characterized by a magnetic island structure with a chain of $X$ and $O$ points and an organization of the fluid motion in convection-like cells or rolls. We note here that even though with increasing Reynolds numbers the equilibrium first becomes unstable to two-dimensional perturbations, the new final states might be three-dimensional (already after the primary bifurcation). This problem is the subject of ongoing 
studies.

\section{ACKNOWLEDGMENTS}

J. S. wishes to thank Prof. J. Kurths, University of Potsdam, for making available financial support during a phase of this work. 


\section{REFERENCES}

1. D. Biskamp, Nonlinear Magnetohydrodynamics (Cambridge University Press, Cambridge, England, 1993).

2. R. J. Goldston and P. H. Rutherford, Introduction to Plasma Physics (Institute of Physics Publishing, Bristol, 1995).

3. E. R. Priest, Solar Magnetohydrodynamics (D. Reidel Publishing Company, Dordrecht, 1982).

4. H. P. Furth, J. Killeen, and M. N. Rosenbluth, Phys. Fluids 6, 459 (1963).

5. R. B. White, in Handbook of Plasma Physics, edited by M. N. Rosenbluth and R. Z. Sagdeev (North-Holland Publishing Company, Amsterdam, 1983), Vol. 1, pp. 611-676.

6. J. Wesson, Nucl. Fusion 6, 130 (1966).

7. D. Schnack and J. Killeen, Theoretical and Computational Plasma Physics (Int. Atomic Energy Agency, Vienna, 1978), pp. 337-360.

8. R. S. Steinolfson and G. Van Hoven, Phys. Fluids 26, 117 (1983).

9. R. B. Dahlburg, T. A. Zang, D. Montgomery, and M. Y. Hussaini, Proc. Natl. Acad. Sci. USA 80, 5798 (1983).

10. D. Montgomery, Plasma Phys. Control. Fusion 34, 1157 (1992).

11. N. Seehafer, E. Zienicke, and F. Feudel, Phys. Rev. E 54, 2863 (1996).

12. X. Shan, D. Montgomery, and H. Chen, Phys. Rev. A 44, 6800 (1991).

13. T. G. Drazin and W. H. Reid, Hydrodynamic Stability (Cambridge University Press, Cambridge, England, 1981).

14. B. Saramito and E. K. Maschke, in Magnetic Reconnection and Turbulence, 
edited by M. Dubois, D. Gresillon, and M. N. Bussac (Editions de Physique, Orsay, 1985), pp. 89-100.

15. B. Saramito and E. K. Maschke, in Magnetic Turbulence and Transport, edited by P. Hennequin and M. A. Dubois (Editions de Physique, Orsay, 1993), pp. $33-42$.

16. E. Harris, Nuovo Cimento 115, 115 (1962).

17. N. Seehafer and J. Schumacher, Phys. Plasmas 4, 4447 (1997).

18. J. D. Crawford and E. Knobloch, Annu. Rev. Fluid Mech. 23, 341 (1991).

19. D. Montgomery and X. Shan, in Small-Scale Structures in ThreeDimensional Hydrodynamic and Magnetohydrodynamic Turbulence, edited by M. Meneguzzi, A. Pouquet, and P.-L. Sulem (Springer, Berlin, 1995), pp. 241254.

20. B. K. Shivamoggi, Can. J. Phys. 59, 632 (1981).

21. X. Shan and D. Montgomery, Plasma Phys. Control. Fusion 35, 1019 (1993).

22. R. Grauer, Physica D 35, 107 (1989). 


\section{FIGURES}

Fig. 1. Geometry of the magnetohydrodynamic sheet pinch. Arrows in the shaded plane indicate the direction of the equilibrium magnetic field.

Fig. 2. Stability boundaries in the $a-L_{2}$ plane for different values of the Hartmann number $H a$. The parameter $a$ is the current sheet half width of the equilibrium configuration and $L_{2}=2 \pi / k_{2}$ is the wavelength of the perturbation in the $x_{2}$ direction. Asterisks denote calculation with 128 collocation points in the $x_{1}$ direction; the other calculations were made with 64 collocation points.

Fig. 3. Magnetic field lines and velocity stream lines for $a=0.15, \overline{B_{2}^{e}}=0, L_{2}=3$, and $H a=100$. Solid (dashed) velocity stream lines correspond to clockwise (counterclockwise) motion. A mixture of $20 \%$ perturbation $\mathbf{b}$ and $80 \%$ equilibrium field $\mathbf{B}^{e}$ was taken for the magnetic field. The lower left panel shows the undisturbed cross-sheet equilibrium profile $B_{2}^{e}\left(x_{1}\right)$.

Fig. 4. Stability boundaries for different values of $\overline{B_{2}^{e}}$ (see legend in left panel) in the $a-L_{2}$ plane for $H a=10^{3}$ and $H a=10^{4}$. Triangles denote calculated points.

Fig. 5. Magnetic field lines and velocity stream lines for $a=0.15, \overline{B_{2}^{e}}=0.4, L_{2}=6$, and $H a=1000$. The indication of the flow direction and the mixture ratio for the magnetic field are as in Fig. 3. The lower left panel shows the undisturbed cross-sheet equilibrium profile $B_{2}^{e}\left(x_{1}\right)$. 
Fig. 6. Global stability boundary in the $\mathrm{Ha}-\Theta_{0}$ plane, where $\Theta_{0}={\overline{B_{2}^{e}}}^{-1}$, for three cases: (1) $a=0.15$ and $L_{2}=3.0 ;$ (2) $a=0.25$ and $L_{2}=6.0$; (3) $a=0.25$ and $L_{2}=3.0$. Crosses denote calculated points. Points below the dashed line correspond to completely stable equilibria. Diamonds indicate the parameters of the stability curves in Fig. 4. 




Fig. 1; Seehafer 


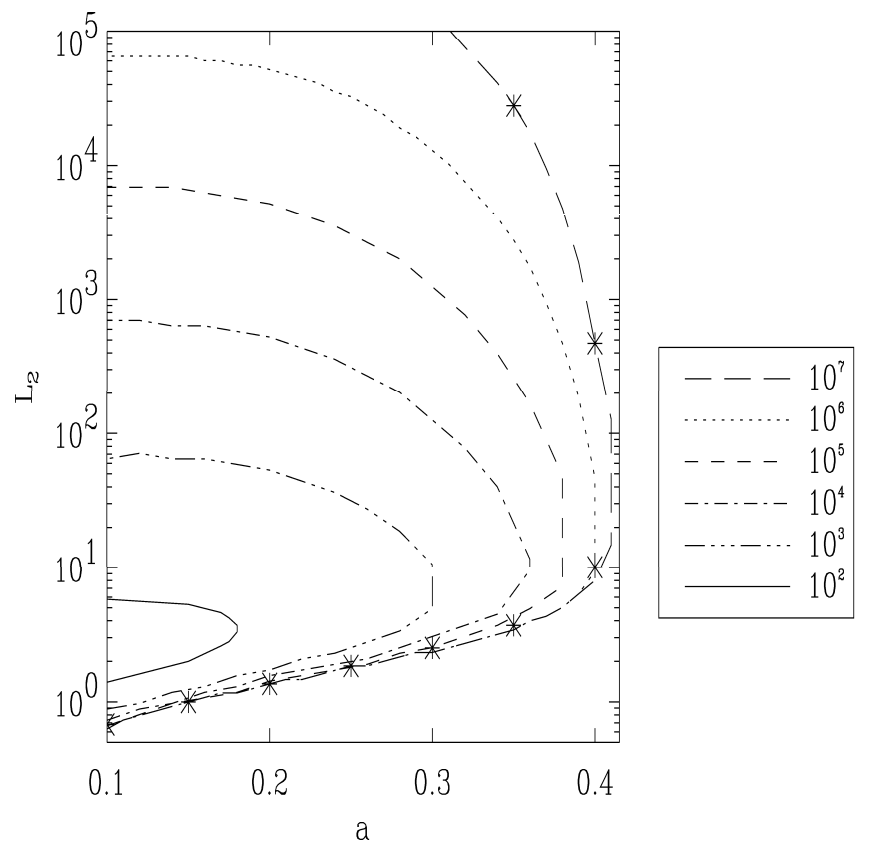

Fig. 2; Seehafer 


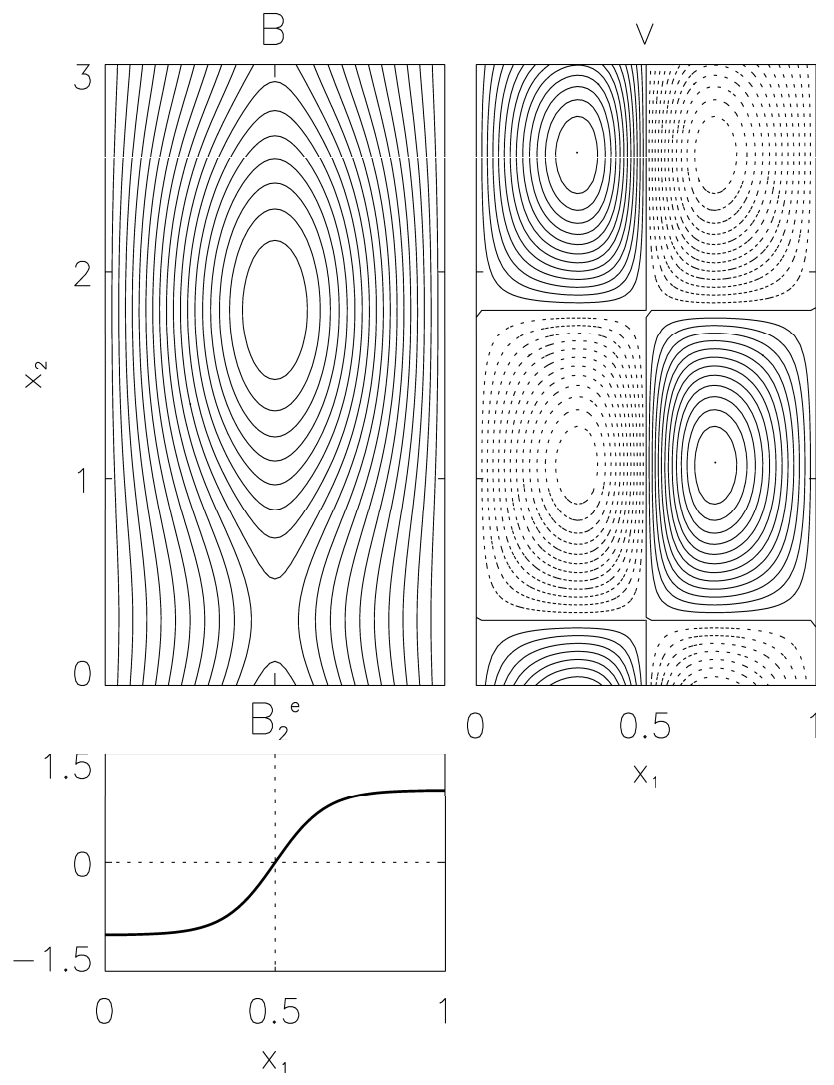

Fig. 3; Seehafer 




Fig. 4; Seehafer 


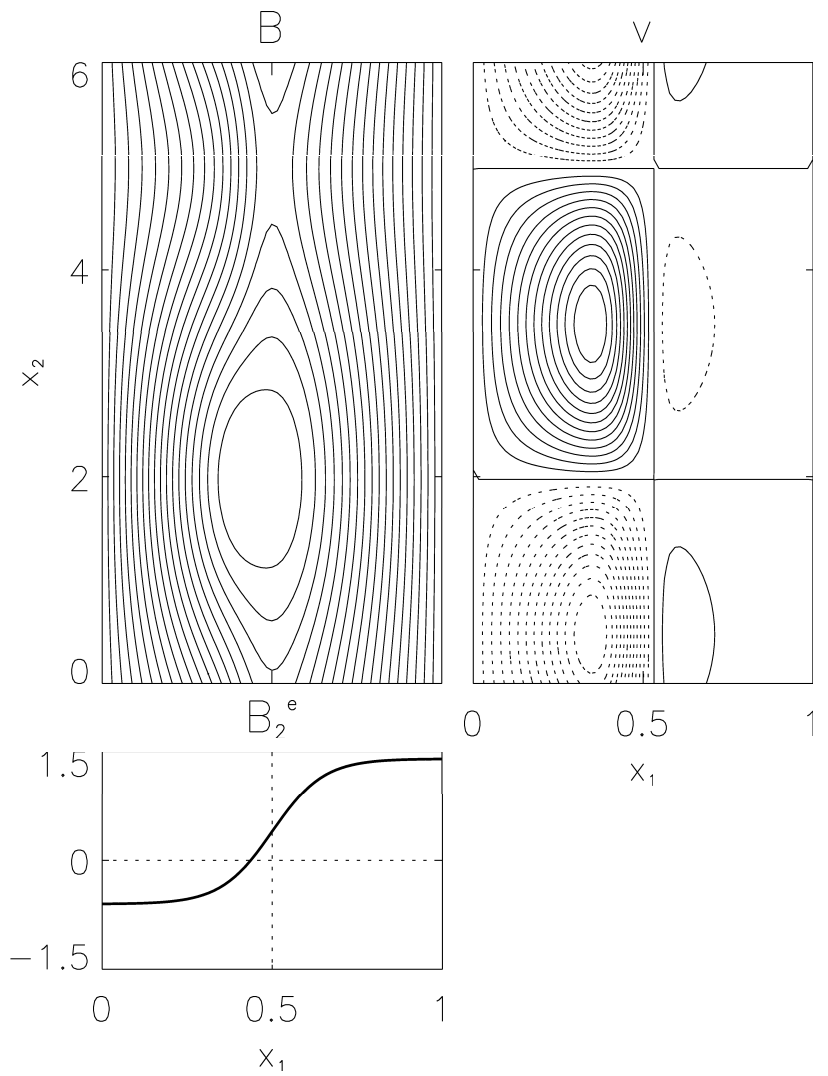

Fig. 5; Seehafer 


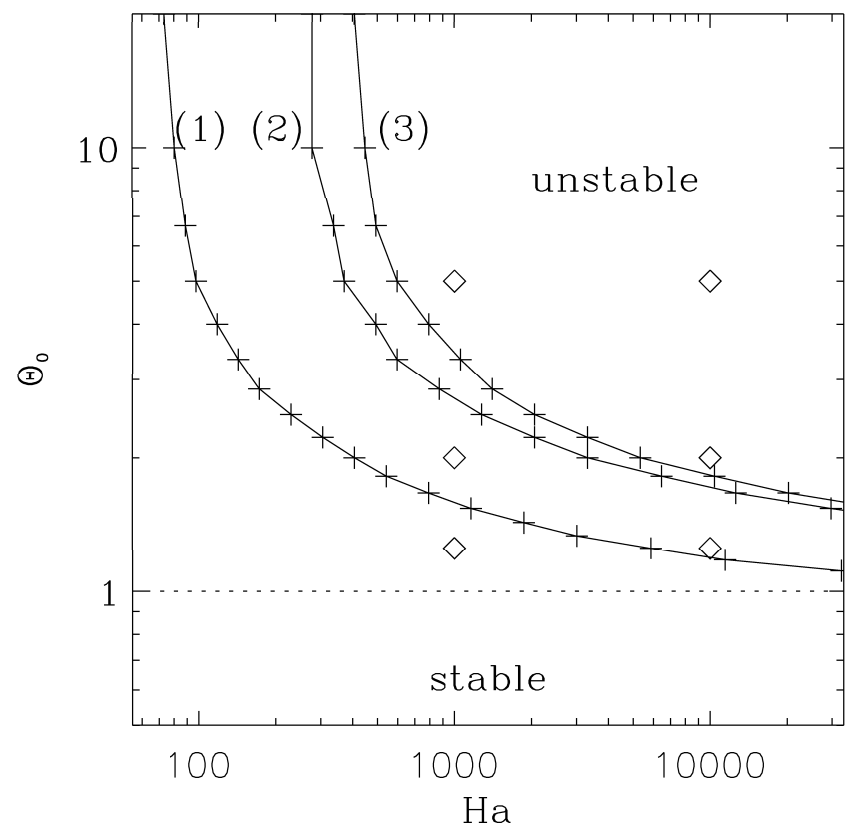

Fig. 6; Seehafer 